\title{
The Local Maximum Clustering Method and Its Application in Microarray Gene Expression Data Analysis
}

\author{
Xiongwu Wu \\ Laboratory of Biophysical Chemistry, National Heart, Lung, and Blood Institute, National Institutes of Health, \\ Bethesda, MD 20892, USA \\ Email:wuxw@nhlbi.nih.gov \\ Yidong Chen \\ National Human Genome Research Institute, National Institutes of Health, Bethesda, MD 20892, USA \\ Email:yidong@nhgri.nih.gov \\ Bernard R. Brooks \\ Laboratory of Biophysical Chemistry, National Heart, Lung, and Blood Institute, National Institutes of Health, \\ Bethesda, MD 20892, USA \\ Email:brb@nih.gov
}

Yan A. Su

Department of Pathology, Loyola University Medical Center, Maywood, IL 60153, USA

Email: ysu2@lumc.edu

Received 28 February 2003; Revised 25 July 2003

\begin{abstract}
An unsupervised data clustering method, called the local maximum clustering (LMC) method, is proposed for identifying clusters in experiment data sets based on research interest. A magnitude property is defined according to research purposes, and data sets are clustered around each local maximum of the magnitude property. By properly defining a magnitude property, this method can overcome many difficulties in microarray data clustering such as reduced projection in similarities, noises, and arbitrary gene distribution. To critically evaluate the performance of this clustering method in comparison with other methods, we designed three model data sets with known cluster distributions and applied the LMC method as well as the hierarchic clustering method, the $K$-mean clustering method, and the self-organized map method to these model data sets. The results show that the LMC method produces the most accurate clustering results. As an example of application, we applied the method to cluster the leukemia samples reported in the microarray study of Golub et al. (1999).
\end{abstract}

Keywords and phrases: data cluster, clustering method, microarray, gene expression, classification, model data sets.

\section{INTRODUCTION}

Data analysis is a key step in obtaining information from large-scale gene expression data. Many analysis methods and algorithms have been developed for the analysis of the gene expression matrix $[1,2,3,4,5,6,7,8,9]$. The clustering of genes for finding coregulated and functionally related groups is particularly interesting in cases where there is a complete set of organism's genes. A reasonable hypothesis is that genes with similar expression profiles, that is, genes that are coexpressed, may have something in common in their regulatory mechanisms, that is, they may be coregulated. Therefore, by clustering together genes with similar expression profiles, one can find groups of potentially coregulated genes and search for putative regulatory signals. So far, many clustering methods have been developed. They can be divided into two categories: supervised and unsupervised methods. This work focuses on unsupervised data clustering. Some widely used methods in this category are the hierarchic clustering method [6], the $K$-mean clustering method [10], and the self-organized map clustering method $[9,11]$.

The clustering of microarray gene expression data typically aims to group genes with similar biological functions or to classify samples with similar gene expression profiles. There are several factors that make the clustering of gene expression data different from data clustering in a general 
sense. First, the "positions" of genes or samples are unknown. That is, where the data points to be clustered locate is unknown. Instead, the relations between data points (genes or samples) are probed by a series of responses (gene expressions). Generally, the correlation of the response series between data points is used as a measure of their similarity. However, because the number of responses is limited and the responses are not independent from each other, the correlation can only provide a reduced description of the similarities between data points. Just like a projection of data points in a high-dimensional space to a low-dimensional space, many data points far apart may be projected together. It often happens that genes that belong to very different categories are clustered together according to gene expression data. Second, there is only a small number of genes presented in a microarray that are relevant to the biological processes under study. All the rest become noises to the analysis, which need to be filtered out based on some criteria before clustering analysis. Third, the genes chosen to array do not necessarily represent the functional distribution. That is, there exist redundant genes of some functions while very few genes exist of some other functions. This may result in the neglect of those less-redundant gene clusters in a clustering analysis. These facts rise difficulties and uncertainties for cluster analysis. Fortunately, a microarray experiment does not attempt to provide accurate cluster information of all genes being arrayed. Instead, besides many other purposes, a microarray experiment is designed to identify and study those groups, which seem to participate in the studied biological process. The complete gene cluster will be the job of many molecular biology experiments as well as other technologies.

With our interest focused on those functional related genes, we need to identify clusters functionally relevant to the biological process of interest. As stated above, clustering methods solely dependent on similarities may suffer from the difficulties of reduced projection, noises, and arbitrary gene distribution and may not be suitable for microarray research purposes. In this work, we present a general approach to clustering a data set based on research interest. A quantity, which is generally called magnitude, is introduced to represent a property of our interest for clustering. The following sections explain in detail the concept and the clustering method, which we call the local maximum clustering (LMC) method. Additionally, for the purpose of comparison, we worked out an approach to quantitatively calculate the agreement between two hierarchic clustering results for the same data set. Using three model systems, we compared this clustering method with several well-known clustering methods. Finally, as an example of application, we applied the method to cluster the leukemia samples reported in the microarray study of Golub et al. [12].

\section{METHODS AND ALGORITHMS}

\subsection{Distances, magnitudes, and clusters}

For a data set with unknown absolute positions, the distance matrix between data points is used to infer their relative po-

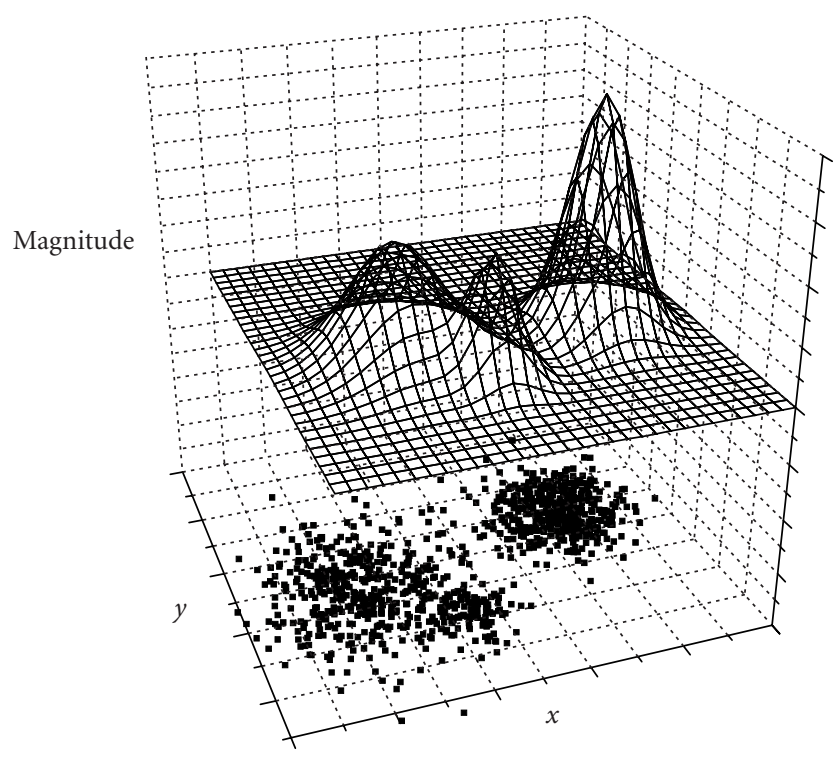

Figure 1: A two-dimensional $(x-y)$ distribution data set with the "magnitude" as the additional dimension.

sitions. For a biologically interesting data set like genes or tissue samples, the distances are not directly measurable. Instead, the responses to a series event are used to estimate the distances or similarity. It is assumed that data points close to each other have similar responses.

For microarray gene expression data, people often use Pearson correlation function to describe the similarity between genes $i$ and $j$ :

$$
C_{i j}=\frac{1}{n} \sum_{k=1}^{n}\left(\frac{X_{i k}-\bar{X}_{i}}{\sigma_{i}}\right)\left(\frac{X_{j k}-\bar{X}_{j}}{\sigma_{j}}\right),
$$

where $X_{i}=\left(X_{i k}\right)_{n}, k=1, \ldots, n$, represents the data point of gene $i$, which consists of $n$ responses, $X_{i k}$ is the $k$ th response of gene $i, \bar{X}_{i}$ is the average value of $X_{i}, \bar{X}_{i}=(1 / n) \sum_{k=1}^{n} X_{i k}$, and $\sigma_{i}$ is the standard deviation of $X_{i}, \sigma_{i}=\sqrt{X_{i}^{2}}-\bar{X}_{i}^{2}$.

From (1), we can see that $C_{i j}$ ranges from -1 to 1 , with 1 representing identical responses between genes $i$ and $j$ and -1 the opposite responses. The distance between a pair of genes is often expressed as the following function:

$$
r_{i j}=1-C_{i j} .
$$

We introduce a quantity called magnitude to represent our research interest. This magnitude is introduced as an additional dimension to the distribution space. We image a set of data points distributed on $x-y$ plan, a two-dimensional space, the magnitude will be an additional dimension, $z$ dimension (Figure 1). Usually, a cluster is a collection of data points that are more similar to each other than to data points in different clusters. Clusters of this type are characterized by a magnitude of the local densities with each cluster representing a high-density region. Here, the local density is the 
magnitude used to define clusters. We should keep in mind that the magnitude property can be properties other than density; it can be gene expression levels or gene differential expressions as described later. As can be seen from Figure 1, each cluster is represented by a peak on the magnitude surface. Obviously, clusters in a data set can be found out by identifying peaks on the magnitude surface. Because clusters are peaks on the magnitude surface, the number and size of clusters depend only on the surface shape.

Current existing clustering methods like the hierarchic clustering method do not explicitly use the magnitude property. These clustering methods assume clusters locate at highdensity areas of a distribution. In other words, these clustering methods implicitly use distribution density as the magnitude of clustering.

The choosing of the magnitude property determines what we want to be the cluster centers. If we want clusters to center at high-density areas, using distribution density would be a natural choice for the magnitude. A simple distribution density can be calculated as

$$
M_{i}=\sum_{j=1}^{n} \delta\left(r_{i j}\right)
$$

where $\delta\left(r_{i j}\right)$ is a step function:

$$
\delta\left(r_{i j}\right)= \begin{cases}1 & r_{i j} \leq d \\ 0 & r_{i j}>d\end{cases}
$$

Equation (3) indicates the magnitude of data point $i$ and $M_{i}$ is equal to the number of data points within distance $d$ from data point $i$. A smaller $d$ will result in a more accurate local density but a larger statistic error. To make the magnitude smooth, an alternative function can be used for $\delta\left(r_{i j}\right)$ :

$$
\delta\left(r_{i j}\right)=\exp \left(-\frac{r_{i j}^{2}}{2 d^{2}}\right) .
$$

For microarray studies, directly clustering genes based on density may result in misleading results. The main reason is that we do not know the real "positions" of the genes. The relative similarities between genes are probed by their responses to an often very limited number of samples. The similarity obtained this way is a reduced projection of "real" similarities, and many very different functional genes may respond similarly in the limited sample set. Therefore, the densities estimated from the response data are not reliable and change from experiment to experiment. Further, the correlation function captures similarity of the shapes of two expression profiles, but it ignores the strength of their responses. Some noises in response measurement may cause a nonresponsive gene to be of high correlation with a high-response gene. Another reason is that the genes arrayed in a chip may vary in redundancy, resulting in different density distributions. An extreme case is when a single gene is redundant so many times that they occupy a large portion of an array-a cluster centering at this gene would be created. Additionally, for the thousands of genes arrayed on a gene chip, generally, only a handful of genes show varying expression levels, which we used to probe gene functions. All the rest only show undetectable expressions or simply noises which may result in very high correlation to some genes. Normally, only those genes with significantly varying expression levels can be of meaningfully functional relation, while for the rest we can draw little information from a microarray experiment. Therefore, for a microarray study, a good choice of magnitude would be a quantity measuring the variation of expression levels as in

$$
M_{i}=\delta^{2}\left(\ln R_{i}\right)=\frac{1}{n} \sum_{j=1}^{n}\left(\ln R_{i j}\right)^{2}-\left(\frac{1}{n} \sum_{j=1}^{n} \ln R_{i j}\right)^{2},
$$

where $R_{i}$ is the expression ratio between sample and control and $n$ is the number of samples for each gene. Equation (6) is a magnitude defined as the differential expression of genes. By this definition, the clusters are always centered at highdifferential expression genes. Because this paper focuses on the presentation and evaluation of the local maximum clustering method, we will not discuss the application of (6) in identifying high-response gene clusters. This equation is presented here only to illustrate the idea of the magnitude properties.

\subsection{The local maximum clustering method}

Two types of properties characterize the data points: magnitude of each data point and distance (or similarity) between a pair of data points. We define a cluster as a peak on the magnitude surface. Therefore, we can cluster a data set by identifying peaks on the magnitude surface.

There are many approaches to identifying peaks on a surface. Here, in this work, we use a method called the local maximum method to identify peaks. Identification of peaks on a surface can be done by searching for the local maximum point around each data point. Assume there is a data set of $N$ data points to be clustered. The local maximum of a data point $i$ is the data point whose magnitude is the maximum among all the data points within a certain distance from the data point $i$. A peak has the maximum magnitude in its local area, therefore, its local maximum is itself. By identifying all data points whose local maximum points are themselves, we can locate all the peaks on the magnitude surface. The distance used to define the local area is called resolution. The number of peaks on a magnitude surface depends on the shape of the surface and the size of resolution. After the peaks are identified, all data points can be assigned into these peaks according to their local maximum points in the way that a data point belongs to the same peak as its local maximum point.

Figure 2 shows a one-dimensional distribution of a data set along the $x$-axis. The $y$-axis is the magnitude of the data set. The peaks represent cluster centers depending on the resolution $r_{0}$. Clusters can be identified by searching for the peaks in the distribution, and all data points can be clustered into these peaks according to the local maximums of each data point. Assume that $r_{1}, r_{3}$, and $r_{4}$ are the distances from peaks 1,3 , and 4 to their nearest equal-magnitude neighbor points. With a resolution $r_{0}<r_{3}$, four peaks, $1,2,3$, and 4 can be identified as the local maximum points of themselves. All 


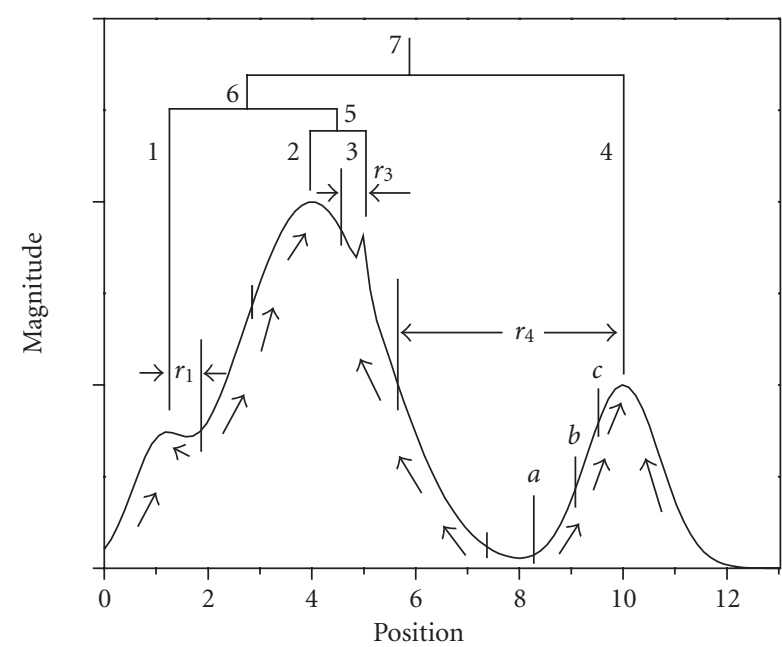

FIgURE 2: Clustering a data set based on the local maximum of its magnitude. There are 4 peaks, $1,2,3$, and 4 ; and $r_{1}, r_{3}$, and $r_{4}$ are the distances from peaks 1,3 , and 4 to their nearest equal magnitude neighbor points. Assume $r_{3}<r_{1}<r_{4}$.

data points can be clustered into these four peaks according to their local maximum points. For example, for data point $a$, if data point $b$ is the one that has the maximum magnitude in all data points within $r_{0}$ from $a$, we say $b$ is the local maximum point of $a$. Point $a$ will belong to the same peak as point $b$. Similarly, point $b$ belongs to the same peak as its local maximum point $c$ and point $c$ belongs to peak 4 . Therefore, points $a, b$, and $c$ all belong to peak 4 .

Obviously, resolution $r_{0}$ plays a crucial role in identifying peaks. For each peak $p$, we define its resolution limit $r_{p}$ as the longest distance within which peak $p$ has the maximum magnitude. For a given resolution $r_{0}$, a peak $p$ will be identified as a cluster center if $r_{p}>r_{0}$. As shown in Figure 2, there are four peaks, $1,2,3$, and 4 . If $r_{0}>r_{1}$, peak 1 will not be identified and, together with all its neighbors, will be assigned to cluster 2 . Similarly, cluster 3 or 4 can only be identified when $r_{0}<r_{3}$ or $r_{0}<r_{4}$, respectively.

The peaks identified can be further clustered to produce a hierarchic cluster structure. For the example shown in Figure 2, if we assume that $r_{4}>r_{1}>r_{3}$, by using $r_{0}<r_{3}$, we can get four clusters, while, using $r_{1}>r_{0}>r_{3}$, clusters 2 and 3 merge to cluster 5 at peak 2, with $r_{4}>r_{0}>r_{1}$, clusters 1 and 5 merge into cluster 6 at peak 2, and with $r_{0}>r_{4}$, all clusters merge into a single cluster at peak 2 .

The algorithm of the LMC method is described by the following steps.

(i) For a data set $\{i\}, i=1,2, \ldots, N$, calculate the distances between data points $\left\{r_{i j}\right\}$ using (1) and (2). From the distance matrix, calculate the magnitude of each data point $\{M(i)\}$ using (5).

(ii) Set resolution $r_{0}=\min \left\{r_{i j}\right\}+\delta r, i \neq j$. Here, $\delta r$ is the resolution increment. Typically, set $\delta r=0.01$.

(iii) Search for the local maximum point $L(i)$ for each data point $i$. For all $j$, with $r_{i j}<r_{0}$, there is $M(L(i)) \geq M(j)$. (iv) Identify peak centers $\{p\}$, where $L(p)=p$. Each peak represents the center of a cluster.

(v) Assign each data point $i$ to the same cluster as its local maximum point $L(i)$.

(vi) If there is more than one cluster, generate higherlevel clusters from the peak point data set $\{p\}, p=$ $1,2, \ldots, n_{p}$, following steps (ii), (iii), (iv), and (v).

\subsection{Comparison of hierarchic clusters}

For the same data set, different clustering methods may produce different clusters. It is, in general, a nontrivial task to compare different clustering results of the same data set and many efforts have been made for such clustering comparison (e.g., [13]). For hierarchic clustering, comparison is more challenging because a hierarchic cluster is a cluster of clusters. To quantitatively compare hierarchic clusters from different methods, we define the following agreement function to describe the agreement between hierarchic clustering results.

We use $\left\{H_{1}\right\}$ and $\left\{H_{2}\right\}$ to represent two hierarchic clustering results for the same data set. In the following discussions, $N_{1}$ and $N_{2}$ are the numbers of clusters in $\left\{H_{1}\right\}$ and $\left\{H_{2}\right\}$, respectively, $n_{1 i}$ and $n_{2 j}$ represent the data point numbers in cluster $i$ of $\left\{H_{1}\right\}$ and cluster $j$ of $\left\{H_{2}\right\}$, respectively, and $m_{i j}$ is the number of data points existing both in cluster $i$ of $\left\{H_{1}\right\}$ and in cluster $j$ of $\left\{H_{2}\right\}$. Therefore, $2 m_{i j} /\left(n_{1 i}+n_{2 j}\right)$ represents how well the two clusters, cluster $i$ of $\left\{H_{1}\right\}$ and cluster $j$ of $\left\{\mathrm{H}_{2}\right\}$, are similar to each other. A value of 1 indicates they are identical and a value of 0 indicates they are completely different. We use $M_{1 i}\left(\left\{H_{2}\right\}\right)$ to describe how well cluster $i$ of $\left\{H_{1}\right\}$ is clustered in $\left\{H_{2}\right\}$. We call $M_{1 i}\left(\left\{H_{2}\right\}\right)$ the match of $\left\{H_{1}\right\}$ to $\left\{H_{2}\right\}$ in cluster $i$. Similarly, the match of $\left\{H_{2}\right\}$ to $\left\{H_{1}\right\}$ in cluster $j$ is denoted as $M_{2 j}\left(\left\{H_{1}\right\}\right)$, which describes how well cluster $j$ of $\left\{H_{2}\right\}$ is clustered in $\left\{H_{1}\right\}$. They are calculated using the following equations:

$$
\begin{aligned}
& M_{1 i}\left(\left\{H_{2}\right\}\right)=\max _{j \in N_{2}}\left\{\frac{2 m_{i j}}{n_{1 i}+n_{2 j}}\right\}, \\
& M_{2 j}\left(\left\{H_{1}\right\}\right)=\max _{i \in N_{1}}\left\{\frac{2 m_{i j}}{n_{1 i}+n_{2 j}}\right\} .
\end{aligned}
$$

Equations (7) mean that the match of $\left\{H_{1}\right\}$ to $\left\{H_{2}\right\}$ in a cluster is the highest similarity between this cluster and any cluster of $\left\{\mathrm{H}_{2}\right\}$.

We use the agreement $A\left(\left\{H_{1}\right\},\left\{H_{2}\right\}\right)$ to describe the overall similarity between two clustering results, which is a weighted average of all cluster matches, as

$$
\begin{aligned}
A\left(\left\{H_{1}\right\},\left\{H_{2}\right\}\right)= & \frac{1}{2 \sum_{i=1}^{N_{1}} n_{1 i}} \sum_{i=1}^{N_{1}} n_{1 i} M_{1 i}\left(\left\{H_{2}\right\}\right) \\
& +\frac{1}{2 \sum_{j=1}^{N_{2}} n_{2 j}} \sum_{j=1}^{N_{2}} n_{2 j} M_{2 j}\left(\left\{H_{1}\right\}\right) .
\end{aligned}
$$

To further illustrate the definition of the agreement and matches, we show an example of two hierarchic clustering results in Figures $3 \mathrm{a}$ and $3 \mathrm{~b}$. These two hierarchic clustering results, $\left\{H_{A}\right\}$ and $\left\{H_{B}\right\}$, are for the same data set of 1000 


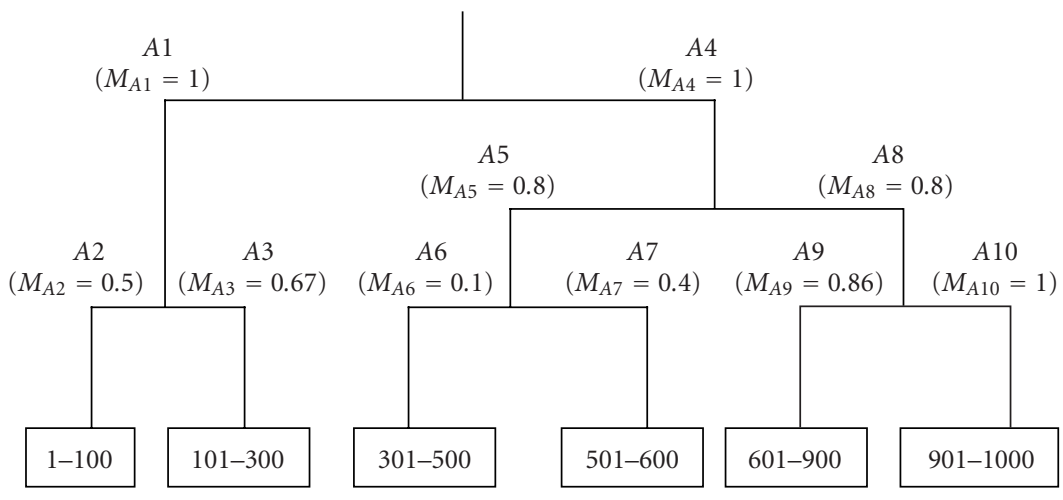

(a)

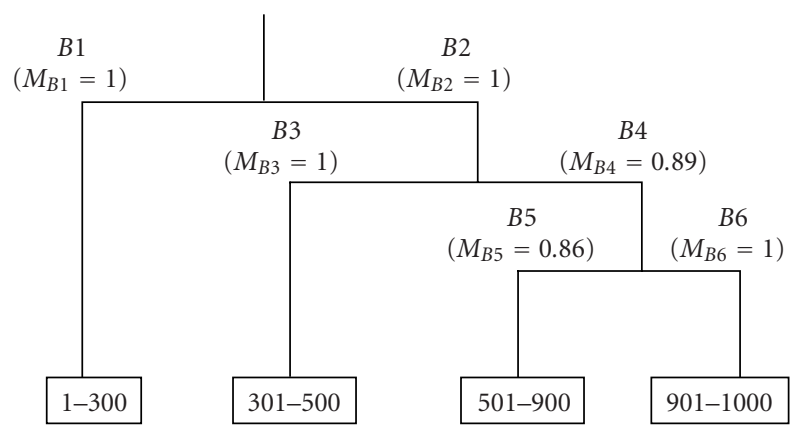

(b)

FIgURE 3: (a) The hierarchic clustering structure $\left\{H_{A}\right\}$ with 10 clusters; the match of each cluster to the cluster structure $\left\{H_{B}\right\}$ are labeled in parentheses; (b) the hierarchic cluster structure $\left\{H_{B}\right\}$ with 6 clusters; the match of each cluster to the cluster structure $\left\{H_{A}\right\}$ are labeled in parentheses.

data points. The hierarchic clustering structure $\left\{H_{A}\right\}$ has 10 clusters and $\left\{H_{B}\right\}$ has 6 clusters. Clusters $A 1, A 4$, and $A 10$ of $\left\{H_{A}\right\}$ have the same data points as clusters $B 1, B 2$, and $B 6$ of $\left\{H_{B}\right\}$, respectively. Therefore, their matches are 1 no matter how different their subclusters are. The matches of clusters are calculated according to (7) and are labeled in the figures. The agreement between $\left\{H_{A}\right\}$ and $\left\{H_{B}\right\}$ can be calculated using (8) as follows:

$$
\begin{aligned}
A\left(\left\{H_{A}\right\},\left\{H_{B}\right\}\right) & \\
= & \frac{\sum_{i=1}^{10} n_{A i} M_{A i}}{2 \sum_{i=1}^{10} n_{A i}}+\frac{\sum_{j=1}^{6} n_{B j} M_{B j}}{2 \sum_{j=1}^{6} n_{B j}} \\
= & \frac{300 \times 1+100 \times 0.5+200 \times 0.67+700 \times 1+300 \times 0.8+200 \times 0.1+100 \times 0.4+400 \times 0.8+300 \times 0.86+100 \times 1}{2(300+100+200+700+300+200+100+400+300+100)} \\
& +\frac{300 \times 1+700 \times 1+200 \times 1+500 \times 0.89+400 \times 0.86+100 \times 1}{2(300+700+200+500+400+100)} \\
= & 0.400+0.475 \\
= & 0.875 .
\end{aligned}
$$


TABLE 1: The possibility parameters used to generate the three model systems. Each model has 6 clusters. The parameters $\left(h_{i}, w_{i}\right)$ represent the height and width of cluster $i$ in the possibility distribution in (10).

\begin{tabular}{c|cccccc}
\hline Model & $\left(h_{1}, w_{1}\right)$ & $\left(h_{2}, w_{2}\right)$ & $\left(h_{3}, w_{3}\right)$ & $\left(h_{4}, w_{4}\right)$ & $\left(h_{5}, w_{5}\right)$ & $\left(h_{6}, w_{6}\right)$ \\
\hline 1 & $(1,0.05)$ & $(1,0.02)$ & $(1,0.02)$ & $(1,0.05)$ & $(1,0.02)$ & $(1,0.02)$ \\
2 & $(1,0.10)$ & $(1,0.005)$ & $(1,0.05)$ & $(1,0.10)$ & $(1,0.005)$ & $(1,0.10)$ \\
3 & $(1,0.10)$ & $(2,0.005)$ & $(3,0.05)$ & $(4,0.10)$ & $(5,0.005)$ & $(6,0.10)$ \\
\hline
\end{tabular}

\section{RESULTS AND DISCUSSIONS}

The LMC method has several features. First, it is an unsupervised clustering method. The clustering result depends on the data set itself. Second, it allows magnitude properties to be used to identify clusters of interest. Third, it automatically produces a hierarchic cluster structure with a minimum amount of input. In this work, we designed three model systems with known cluster distributions to evaluate the performance of the LMC method and compare it with other methods. Finally, as an example of application, we use this method to cluster the leukemia samples reported by Golub et al. [12] and compare the result with experimental classification.

\subsection{The model systems}

Model systems with known cluster distributions have often been used in method development. The model systems used here are designed to mimic microarray gene expression data in the way that each data point is a response series of expression values, and the distance or similarity between data points is measured by their correlation function. It is the correlation function that determines the distance between data points and the actual number of expression values in a response series, which does not affect the clustering results; for simplicity and convenience of data generation and analysis, we use only three expression values for each response series, namely, $x, y$, and $z$. The response series of gene $i$ is represented by $\left(x_{i}, y_{i}, z_{i}\right)$. The correlation function and distance between gene $i$ and gene $j$ is calculated according to (1) and (2) with $n=3$.

The model systems are designed to have 6 clusters with cluster centers at $\left(X_{j}, Y_{j}, Z_{j}\right), j=1,2,3,4,5$, and 6 . We use the following possibility distribution to generate the expression data of 1000 genes $\left(x_{i}, y_{i}, z_{i}\right), i=1,2, \ldots, 1000$ :

$$
\rho\left(x_{i}, y_{i}, z_{i}\right)=\sum_{j=1}^{6} h_{j} \exp \left(-\frac{\left(1-C_{i j}\right)^{2}}{2 w_{j}^{2}}\right),
$$

where $\rho\left(x_{i}, y_{i}, z_{i}\right)$ represents the possibility function to have a gene with a response series of $\rho\left(x_{i}, y_{i}, z_{i}\right)$, and $h_{j}$ and $w_{j}$ are the height and width of cluster $j$. The six cluster centers are genes with the following response series:

(i) $(-\sqrt{2} / 2,0, \sqrt{2} / 2)$;

(ii) $(-\sqrt{2} / 2, \sqrt{2} / 2,0)$;

(iii) $(-1 / \sqrt{6}, 2 / \sqrt{6},-1 / \sqrt{6})$;

(iv) $(0,-\sqrt{2} / 2, \sqrt{2} / 2)$;

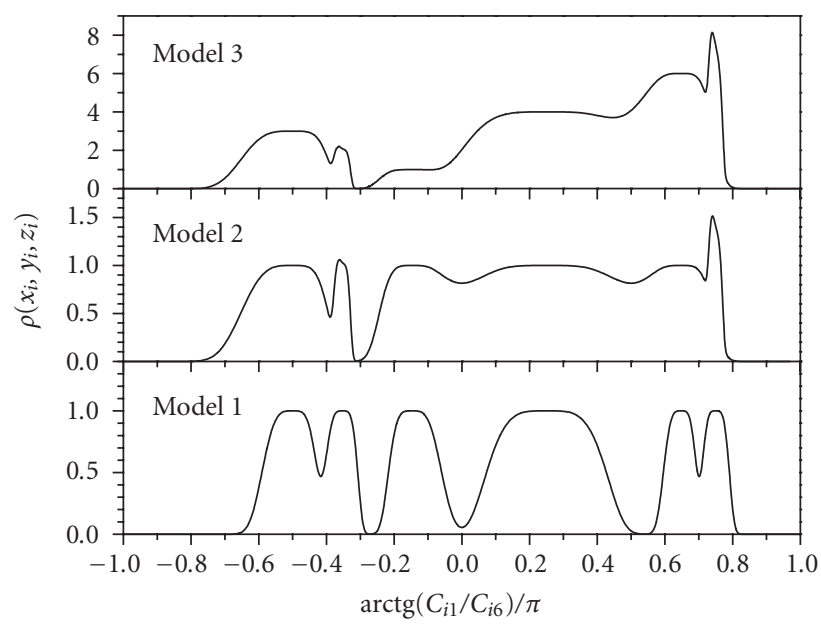

Figure 4: Data distribution in the three model data sets. The function $\operatorname{arctg}\left(C_{i 1} / C_{i 6}\right) / \pi$ is used for the $x$-axis to show all six clusters without overlapping. Here, $C_{i 1}$ and $C_{i 6}$ are the correlations of data point $i$ with the centers of clusters 1 and 6, respectively. For each model, 1000 data points are generated.

(v) $(2 / \sqrt{6},-1 / \sqrt{6},-1 / \sqrt{6})$;

(vi) $(\sqrt{2} / 2,-\sqrt{2} / 2,0)$.

The correlation matrix between these centering genes is

$$
\left[C_{i j}\right]_{6 \times 6}=\left(\begin{array}{ccccccc}
1 & \frac{1}{2} & 0 & \frac{1}{2} & -\frac{\sqrt{3}}{2} & -\frac{1}{2} \\
\frac{1}{2} & 1 & \frac{\sqrt{3}}{2} & -\frac{1}{2} & -\frac{\sqrt{3}}{2} & -1 \\
0 & \frac{\sqrt{3}}{2} & 1 & -\frac{\sqrt{3}}{2} & -\frac{1}{2} & -\frac{\sqrt{3}}{2} \\
\frac{1}{2} & -\frac{1}{2} & -\frac{\sqrt{3}}{2} & 1 & 0 & \frac{1}{2} \\
-\frac{\sqrt{3}}{2} & -\frac{\sqrt{3}}{2} & -\frac{1}{2} & 0 & 1 & \frac{\sqrt{3}}{2} \\
-\frac{1}{2} & -1 & -\frac{\sqrt{3}}{2} & \frac{1}{2} & \frac{\sqrt{3}}{2} & 1
\end{array}\right) .
$$

Three model data sets, each has 1000 data points, are generated using the parameters listed in Table 1. Their distributions are shown in Figure 4. The clusters are separated 


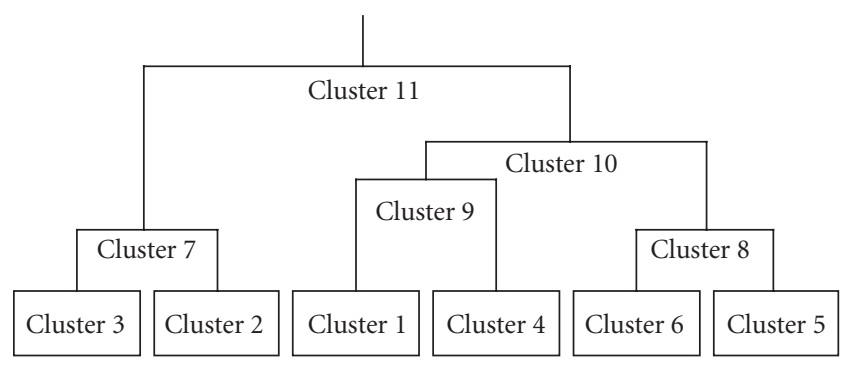

Figure 5: The hierarchic cluster structure of the model data sets.

TABLE 2: Comparison of the clustering results of different methods. The letters L, H, K, and S stand for the LMC method, the hierarchic clustering method, the $K$-mean clustering method, and the self-organization map clustering method, respectively.

\begin{tabular}{|c|c|c|c|c|c|c|c|c|c|c|c|c|c|}
\hline & \multirow[b]{2}{*}{ Clusters } & \multicolumn{4}{|c|}{ Model 1} & \multicolumn{4}{|c|}{ Model 2} & \multicolumn{4}{|c|}{ Model 3} \\
\hline & & $\mathrm{L}$ & $\mathrm{H}$ & $\mathrm{K}$ & $S$ & $\mathrm{~L}$ & $\mathrm{H}$ & $\mathrm{K}$ & $S$ & $\mathrm{~L}$ & $\mathrm{H}$ & $\mathrm{K}$ & $S$ \\
\hline \multirow{10}{*}{$\begin{array}{l}\text { Matches to the } \\
\text { models }(\%)\end{array}$} & 1 & 99.7 & 97.2 & 68.0 & 68.0 & 87.8 & 87.8 & 87.8 & 87.2 & 89.8 & 85.2 & 85.0 & 85.2 \\
\hline & 2 & 99.2 & 96.8 & 65.2 & 65.2 & 98.0 & 94.6 & 35.6 & 36.0 & 78.2 & 85.8 & 41.0 & 40.8 \\
\hline & 3 & 99.6 & 99.6 & 69.7 & 88.3 & 94.4 & 80.8 & 71.1 & 67.4 & 91.8 & 43.8 & 95.8 & 70.7 \\
\hline & 4 & 99.8 & 99.8 & 68.8 & 77.4 & 69.5 & 67.5 & 77.5 & 72.3 & 89.0 & 78.8 & 71.8 & 70.8 \\
\hline & 5 & 98.1 & 99.2 & 62.3 & 63.1 & 80.1 & 76.9 & 76.2 & 80.4 & 88.4 & 76.6 & 78.8 & 65.4 \\
\hline & 6 & 98.4 & 98.4 & 70.6 & 70.2 & 92.5 & 96.9 & 70.0 & 45.2 & 91.1 & 96.2 & 75.8 & 55.8 \\
\hline & 7 & 99.8 & 99.8 & - & - & 99.8 & 99.7 & - & - & 99.8 & 99.8 & - & - \\
\hline & 8 & 100 & 100 & - & - & 97.2 & 95.0 & - & - & 95.1 & 94.4 & - & - \\
\hline & 9 & 99.8 & 99.8 & - & - & 98.4 & 82.8 & - & - & 95.0 & 94.4 & - & - \\
\hline & 10 & 100 & 76.8 & 一 & - & 100 & 100 & 一 & - & 100 & 100 & - & - \\
\hline \multicolumn{2}{|c|}{ Overall agreement (\%) } & 96.9 & 69.4 & 76.2 & 81.0 & 88.5 & 65.1 & 75.3 & 76.0 & 89.5 & 67.2 & 79.5 & 72.9 \\
\hline
\end{tabular}

by minimums between peaks, and the data points can be accurately assigned to their clusters. As can be seen (Figure 4) in model 1, the six clusters have equal heights and are clearly separated from each other, while in model 2, clusters 1, 3, 4, and 5 are much broader, and in model 3, their heights are different. These three model data sets present some typical cases that a clustering method would deal with.

Based on the correlations between the clusters, (11), these model data sets have a hierarchic cluster structure as shown in Figure 5. The whole data set belongs to a single cluster 11, which is split into two clusters, 7 and 10 . Cluster 7 is divided into clusters 2 and 3. Cluster 10 is further divided into cluster 9, which consists of clusters 1 and 4 , and cluster 8 , which consists of clusters 5 and 6 .

We applied the LMC method (L), the hierarchic clustering method [6] $(\mathrm{H})$, the $K$-mean clustering method [10] $(\mathrm{K})$, and the self-organized map clustering method [11] (S) to these three model data sets. The LMC method, as well as the hierarchic clustering method, produces a hierarchic cluster structure. The $K$-mean and the self-organized map methods require a predefined cluster number prior to clustering. For comparison purpose, we set the cluster number to 6 when performing clustering using the $K$-mean and the self-organized map method, and only compare the agreement between the clustering results with the bottom 6 clusters of the model data sets. Table 2 listed the matches and agreements between the results from the four clustering methods and the known clusters of the model data sets.

Comparing the matches and agreements between the clustering results and the known clusters of the model data sets, we can see clearly that the LMC method produces the most accurate result. The hierarchic clustering method produces many tree structures, within which there exist good matches to the clusters in the models. Because it produces too many trees, the agreement between the model and result from the hierarchic method is low. The $K$-mean and the self-organized map methods produce worse matches to the clusters in the models than the LMC and the hierarchic clustering methods.

\subsection{An application to microarray gene expression data}

Application of the LMC method to gene expression data is straightforward. As an example of the application, we applied this method to cluster the 72 samples collected by Golub et 
TABLE 3: Classification of the acute lymphoblastic leukemia (ALL) and acute myeloid leukemia (AML) samples [12].

\begin{tabular}{|c|c|c|c|c|c|c|c|c|c|}
\hline \multicolumn{4}{|c|}{ Cluster levels } & \multirow{2}{*}{ Samples } & \multirow{2}{*}{ Type } & \multirow{2}{*}{ Source } & \multirow{2}{*}{ Lineage } & \multirow{2}{*}{ FAB } & \multirow{2}{*}{ Sex } \\
\hline 1 & 2 & 3 & 4 & & & & & & \\
\hline \multirow{47}{*}{$A$} & \multirow{41}{*}{$A 1$} & \multirow{33}{*}{$A 11$} & \multirow{4}{*}{$A 111$} & 4 & ALL & $\mathrm{BM}$ & B-cell & - & - \\
\hline & & & & 20 & ALL & $\mathrm{BM}$ & B-cell & - & - \\
\hline & & & & 5 & ALL & $\mathrm{BM}$ & B-cell & - & - \\
\hline & & & & 19 & ALL & $\mathrm{BM}$ & B-cell & - & - \\
\hline & & & \multirow{19}{*}{$A 112$} & 46 & ALL & $\mathrm{BM}$ & B-cell & - & $\mathrm{F}$ \\
\hline & & & & 12 & ALL & $\mathrm{BM}$ & B-cell & - & $\mathrm{F}$ \\
\hline & & & & 42 & ALL & $\mathrm{BM}$ & B-cell & - & $\mathrm{F}$ \\
\hline & & & & 48 & ALL & $\mathrm{BM}$ & B-cell & - & $\mathrm{F}$ \\
\hline & & & & 7 & ALL & $\mathrm{BM}$ & B-cell & - & $\mathrm{F}$ \\
\hline & & & & 59 & ALL & $\mathrm{BM}$ & B-cell & - & $\mathrm{F}$ \\
\hline & & & & 8 & ALL & $\mathrm{BM}$ & B-cell & - & $\mathrm{F}$ \\
\hline & & & & 15 & ALL & $\mathrm{BM}$ & B-cell & - & $\mathrm{F}$ \\
\hline & & & & 18 & ALL & $\mathrm{BM}$ & B-cell & - & $\mathrm{F}$ \\
\hline & & & & 43 & ALL & BM & B-cell & - & $\mathrm{F}$ \\
\hline & & & & 56 & ALL & $\mathrm{BM}$ & B-cell & - & $\mathrm{F}$ \\
\hline & & & & 40 & ALL & $\mathrm{BM}$ & B-cell & - & $\mathrm{F}$ \\
\hline & & & & 44 & ALL & $\mathrm{BM}$ & B-cell & - & $\mathrm{F}$ \\
\hline & & & & 27 & ALL & $\mathrm{BM}$ & B-cell & - & $\mathrm{F}$ \\
\hline & & & & 26 & ALL & $\mathrm{BM}$ & B-cell & - & $\mathrm{F}$ \\
\hline & & & & 55 & ALL & $\mathrm{BM}$ & B-cell & - & $\mathrm{F}$ \\
\hline & & & & 39 & ALL & $\mathrm{BM}$ & B-cell & - & $\mathrm{F}$ \\
\hline & & & & 41 & ALL & $\mathrm{BM}$ & B-cell & - & $\mathrm{F}$ \\
\hline & & & & 13 & ALL & $\mathrm{BM}$ & B-cell & - & $\mathrm{F}$ \\
\hline & & & \multirow{10}{*}{$A 113$} & 17 & ALL & $\mathrm{BM}$ & B-cell & - & M \\
\hline & & & & 16 & ALL & $\mathrm{BM}$ & B-cell & - & M \\
\hline & & & & 21 & ALL & $\mathrm{BM}$ & B-cell & - & M \\
\hline & & & & 45 & ALL & $\mathrm{BM}$ & B-cell & - & M \\
\hline & & & & 22 & ALL & $\mathrm{BM}$ & B-cell & - & M \\
\hline & & & & 25 & ALL & $\mathrm{BM}$ & B-cell & - & M \\
\hline & & & & 24 & ALL & $\mathrm{BM}$ & B-cell & - & M \\
\hline & & & & 47 & ALL & $\mathrm{BM}$ & B-cell & - & M \\
\hline & & & & 1 & ALL & $\mathrm{BM}$ & B-cell & - & M \\
\hline & & & & 49 & ALL & $\mathrm{BM}$ & B-cell & - & M \\
\hline & & \multirow{8}{*}{\multicolumn{2}{|c|}{$A 12$}} & 23 & ALL & $\mathrm{BM}$ & T-cell & - & $\mathrm{M}$ \\
\hline & & & & 10 & ALL & $\mathrm{BM}$ & T-cell & - & M \\
\hline & & & & 3 & ALL & $\mathrm{BM}$ & T-cell & - & M \\
\hline & & & & 11 & ALL & $\mathrm{BM}$ & T-cell & - & M \\
\hline & & & & 2 & ALL & $\mathrm{BM}$ & T-cell & - & M \\
\hline & & & & 6 & ALL & $\mathrm{BM}$ & T-cell & - & M \\
\hline & & & & 14 & ALL & $\mathrm{BM}$ & T-cell & - & M \\
\hline & & & & 9 & ALL & $\mathrm{BM}$ & T-cell & - & $\mathrm{M}$ \\
\hline & \multirow{6}{*}{$A 2$} & \multirow{5}{*}{$A 21$} & \multirow{2}{*}{$A 211$} & 72 & ALL & $\mathrm{PB}$ & B-cell & - & - \\
\hline & & & & 71 & ALL & $\mathrm{PB}$ & B-cell & - & - \\
\hline & & & A212 & 70 & ALL & $\mathrm{PB}$ & B-cell & - & $\mathrm{F}$ \\
\hline & & & \multirow{2}{*}{$A 213$} & 68 & ALL & $\mathrm{PB}$ & B-cell & - & M \\
\hline & & & & 69 & ALL & $\mathrm{PB}$ & B-cell & - & $\mathrm{M}$ \\
\hline & & \multicolumn{2}{|c|}{ A22 } & 67 & ALL & $\mathrm{PB}$ & T-cell & - & M \\
\hline
\end{tabular}


Table 3: Continued.

\begin{tabular}{|c|c|c|c|c|c|c|c|c|c|}
\hline \multicolumn{4}{|c|}{ Cluster levels } & \multirow{2}{*}{ Samples } & \multirow{2}{*}{ Type } & \multirow{2}{*}{ Source } & \multirow{2}{*}{ Lineage } & \multirow{2}{*}{ FAB } & \multirow{2}{*}{ Sex } \\
\hline 1 & 2 & 3 & 4 & & & & & & \\
\hline \multirow{25}{*}{$B$} & \multirow{21}{*}{$B 1$} & \multirow{2}{*}{\multicolumn{2}{|c|}{$B 11$}} & 66 & AML & BM & - & - & M \\
\hline & & & & 65 & AML & $\mathrm{BM}$ & - & - & $\mathrm{M}$ \\
\hline & & \multirow{4}{*}{\multicolumn{2}{|c|}{$B 12$}} & 35 & AML & BM & - & $M 1$ & - \\
\hline & & & & 38 & AML & BM & - & $M 1$ & - \\
\hline & & & & 61 & AML & $\mathrm{BM}$ & - & $M 1$ & - \\
\hline & & & & 32 & AML & BM & - & $M 1$ & - \\
\hline & & \multirow{10}{*}{$B 13$} & \multirow{8}{*}{$B 131$} & 58 & AML & $\mathrm{BM}$ & - & M2 & - \\
\hline & & & & 34 & AML & $\mathrm{BM}$ & - & M2 & - \\
\hline & & & & 28 & AML & $\mathrm{BM}$ & - & M2 & - \\
\hline & & & & 37 & AML & $\mathrm{BM}$ & - & $M 2$ & - \\
\hline & & & & 51 & AML & BM & - & $M 2$ & - \\
\hline & & & & 29 & AML & $\mathrm{BM}$ & - & $M 2$ & - \\
\hline & & & & 33 & AML & BM & - & M2 & - \\
\hline & & & & 53 & AML & BM & - & M2 & - \\
\hline & & & $B 132$ & 57 & AML & $\mathrm{BM}$ & - & $M 2$ & $\mathrm{~F}$ \\
\hline & & & $B 133$ & 60 & AML & BM & - & $M 2$ & M \\
\hline & & \multirow{3}{*}{$B 14$} & \multirow{2}{*}{$B 141$} & 31 & AML & $\mathrm{BM}$ & - & M4 & - \\
\hline & & & & 50 & AML & $\mathrm{BM}$ & - & M4 & - \\
\hline & & & $B 142$ & 54 & AML & $\mathrm{BM}$ & - & M4 & $\mathrm{F}$ \\
\hline & & \multirow{2}{*}{\multicolumn{2}{|c|}{$B 15$}} & 36 & AML & $\mathrm{BM}$ & - & M5 & - \\
\hline & & & & 30 & AML & $\mathrm{BM}$ & - & M5 & - \\
\hline & \multirow{4}{*}{$B 2$} & \multirow{3}{*}{$B 21$} & $B 211$ & 63 & AML & $\mathrm{PB}$ & - & - & $\mathrm{F}$ \\
\hline & & & \multirow{2}{*}{ B212 } & 64 & AML & $\mathrm{PB}$ & - & - & $\mathrm{M}$ \\
\hline & & & & 62 & AML & $\mathrm{PB}$ & - & - & M \\
\hline & & \multicolumn{2}{|c|}{$B 22$} & 52 & AML & $\mathrm{PB}$ & - & M4 & - \\
\hline
\end{tabular}

al. [12] from acute leukemia patients at the time of diagnosis. We choose this data because experimental classification is available for comparison. Table 3 lists the clusters based on experiment classification [12]. The 72 samples contain 47 acute lymphoblastic leukemia (ALL) samples (cluster A) and 25 acute myeloid leukemia (AML) samples (cluster B). These samples are from either bone marrow (BM) (clusters A1 and $\mathrm{B} 1$ ) or peripheral blood (PB) (clusters A2 and B2). The ALL samples fall into two classes: B-lineage ALL (clusters A11 and A21) and T-lineage ALL (clusters A12 and A22), some of which are taken from known sex patients ( $\mathrm{F}$ for female and $\mathrm{M}$ for male). Some of the AML samples have known FAB types, M1-M5.

The whole set of genes are filtered based on expression levels, and 1769 genes with expression levels higher than 20 in all the 72 samples are used for our clustering. That is, for each sample, its response series contains 1769 gene expression values. The logarithms of the gene expression levels are used in correlation function calculation to reduce the noise effect at high expression levels.

We applied the LMC method and the hierarchic clustering method [6] to the 72 samples and compared the results with the experiment clusters listed in Table 3. The magnitude is calculated using (5) so that the cluster centers will be the peaks of local density of data points. Only with this magnitude, the two methods are comparable. The matches of each cluster and the overall agreements of the experimental classification to the clustering results are listed in Table 4 . As can be seen, the ALL samples (cluster $A$ ) can be better clustered by the LMC method $\left(M_{A}(\mathrm{LMC})=0.792\right)$ than by the hierarchic clustering method $\left(M_{A}(\mathrm{HC})=0.784\right)$, while the AML samples can be better described by the hierarchic clustering method $\left(M_{B}(\mathrm{HC})=0.526\right)$ than by LMC method $\left(M_{B}(\mathrm{LMC})=0.521\right)$. Overall, the experimental classification agrees better with the clustering result of the LMC method (the agreement is 0.643 ) than with that of the hierarchic clustering method (the agreement is 0.624 ).

This example shows that the LMC method, like the hierarchic clustering method, can be used for hierarchic clustering of microarray gene expression data. Unlike the hierarchic clustering method, the LMC method has the flexibility to choose magnitude properties, for example, using (6) to cluster high-differential expression genes, which will be the topic of future studies. 
TABle 4: Comparison of the matches and agreements of the experimental classification listed in Table 3 to the clustering results of the LMC method and the HC method.

\begin{tabular}{|c|c|c|}
\hline Clusters & Matches to LMC & Matches to $\mathrm{HC}$ \\
\hline$A$ & 0.7924 & 0.7836 \\
\hline$A 1$ & 0.74 & 0.7252 \\
\hline$A 11$ & 0.6304 & 0.6506 \\
\hline$A 111$ & 0.5 & 0.5 \\
\hline$A 112$ & 0.4358 & 0.4706 \\
\hline$A 113$ & 0.3158 & 0.353 \\
\hline$A 12$ & 0.6666 & 0.6666 \\
\hline$A 2$ & 0.4444 & 0.4 \\
\hline$A 21$ & 0.5 & 0.421 \\
\hline$A 211$ & 0.6666 & 0.3076 \\
\hline$A 213$ & 0.8 & 0.25 \\
\hline$B$ & 0.5208 & 0.5264 \\
\hline$B 1$ & 0.5 & 0.4652 \\
\hline$B 11$ & 0.0816 & 0.25 \\
\hline$B 12$ & 0.1818 & 0.2858 \\
\hline$B 13$ & 0.353 & 0.3076 \\
\hline$B 131$ & 0.4 & 0.3636 \\
\hline$B 14$ & 0.4 & 0.2858 \\
\hline$B 141$ & 0.4444 & 0.3334 \\
\hline$B 15$ & 0.2222 & 0.4 \\
\hline$B 2$ & 0.1066 & 0.1112 \\
\hline$B 21$ & 0.081 & 0.0846 \\
\hline B212 & 0.0548 & 0.0572 \\
\hline Agreement & 0.643 & 0.624 \\
\hline
\end{tabular}

\section{CONCLUSION}

This work proposed the local maximum clustering (LMC) method and evaluated its performance as compared with some typical clustering methods through designed model data sets. This clustering method is an unsupervised one and can generate hierarchic cluster structures with minimum input. It allows a magnitude property of research interest to be chosen for clustering. The comparison using model data sets indicates that the local maximum method can produce more accurate cluster results than the hierarchic, the $K$-mean, and the self-organized map clustering methods. As an example of application, this method is applied to cluster the leukemia samples reported in the microarray study of Golub et al. [12]. The comparison shows that the experimental classification can be better described by the cluster result from the LMC method than by the hierarchic clustering method.

\section{REFERENCES}

[1] A. Brazma and J. Vilo, "Gene expression data analysis," FEBS Letters, vol. 480, no. 1, pp. 17-24, 2000.
[2] M. P. Brown, W. N. Grundy, D. Lin, et al., "Knowledge-based analysis of microarray gene expression data by using support vector machines," Proceedings of the National Academy of Sciences of the USA, vol. 97, no. 1, pp. 262-267, 2000.

[3] J. K. Burgess and Hazelton R. H., "New developments in the analysis of gene expression," Redox Report, vol. 5, no. 2-3, pp. 63-73, 2000.

[4] J. P. Carulli, M. Artinger, P. M. Swain, et al., "High throughput analysis of differential gene expression," Journal of Cellular Biochemistry Supplements, vol. 30-31, pp. 286-296, 1998.

[5] J. M. Claverie, "Computational methods for the identification of differential and coordinated gene expression," Human Molecular Genetics, vol. 8, no. 10, pp. 1821-1832, 1999.

[6] M. B. Eisen, P. T. Spellman, P. O. Brown, and D. Botstein, "Cluster analysis and display of genome-wide expression patterns," Proceedings of the National Academy of Sciences of the USA, vol. 95, no. 25, pp. 14863-14868, 1998.

[7] O. Ermolaeva, M. Rastogi, K. D. Pruitt, et al., "Data management and analysis for gene expression arrays," Nature Genetics, vol. 20, no. 1, pp. 19-23, 1998.

[8] G. Getz, E. Levine, and E. Domany, "Coupled two-way clustering analysis of gene microarray data," Proceedings of the National Academy of Sciences of the USA, vol. 97, no. 22, pp. 12079-12084, 2000.

[9] P. Toronen, M. Kolehmainen, G. Wong, and E. Castren, "Analysis of gene expression data using self-organizing maps," FEBS Letters, vol. 451, no. 2, pp. 142-146, 1999.

[10] S. Tavazoie, J. D. Hughes, M. J. Campbell, R. J. Cho, and G. M. Church, "Systematic determination of genetic network architecture," Nature Genetics, vol. 22, no. 3, pp. 281-285, 1999.

[11] P. Tamayo, D. Slonim, J. Mesirov, et al., "Interpreting patterns of gene expression with self-organizing maps: methods and application to hematopoietic differentiation," Proceedings of the National Academy of Sciences of the USA, vol. 96, no. 6, pp. 2907-2912, 1999.

[12] T. R. Golub, D. K. Slonim, P. Tamayo, et al., "Molecular classification of cancer: class discovery and class prediction by gene expression monitoring," Science, vol. 286, no. 5439, pp. 531537, 1999.

[13] M. Meila, “Comparing clusterings," UW Statistics Tech. Rep. 418, Department of Statistics, University of Washington, Seattle, Wash, USA, 2002, http://www.stat.washington.edu/ $\mathrm{mmp} / \#$ publications/.

Xiongwu Wu received his B.S., M.S., and $\mathrm{Ph} . \mathrm{D}$. degrees in chemical engineering from Tsinghua University, Beijing, China. From 1993 to 1996, he was a Research Fellow in the Cleveland Clinic Foundation, Cleveland, Ohio. Then he worked as a Research Assistant Professor in George Washington University and Georgetown University. He also held an Associate Professor position in Nanjing University of Chemical Technol-

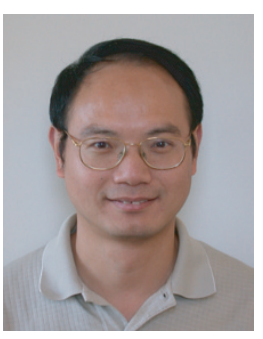
ogy, Nanjing, China. Currently, Dr. Wu is a Staff Scientist at the Laboratory of Biophysical Chemistry, National Heart, Lung, and Blood Institute, National Institutes of Health, Bethesda, Maryland. His research focuses on computational chemistry and biology. His research activities include molecular simulation, protein structure prediction, electron microscopy image processing, and gene expression analysis. He has developed a series of computational methods for efficient and accurate computational studies. 
Yidong Chen received his B.S. and M.S. degrees in electrical engineering from Fudan University, Shanghai, China, in 1983 and 1986, respectively, and his Ph.D. degree in imaging science from Rochester Institute of Technology, Rochester, NY, in 1995. From 1986 to 1988, he joined the Department of Electronic Engineering of Fudan University as an Assistant Professor. From 1988 to 1989, he was a Visiting Scholar in the De-

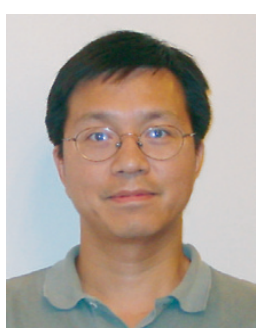
partment of Computer Engineering, Rochester Institute of Technology. From 1995 to 1996, he joined Hewlett Packard Company as a Research Engineer, specialized in digital halftoning and color image processing. Currently, he is a Staff Scientist in the Cancer Genetics Branch of National Human Genome Research Institute, National Institutes of Health, Bethesda, Md, specialized in cDNA microarray bioinformatics and gene expression data analysis. His research interests include statistical data visualization, analysis and management, microarray bioinformatics, genomic signal processing, genetic network modeling, and biomedical image processing.

Bernard R. Brooks obtained his Undergraduate degree in chemistry from the Massachusetts Institute of Technology in 1976 and received his Ph.D. degree in 1979 from the University of California at Berkeley with Professor Henry F. Schaefer. His research efforts at Berkeley focused on the development of methods for electronic structure calculations. In 1980, Dr. Brooks joined Professor Martin Karplus at Harvard Uni-

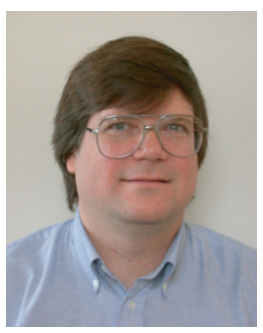
versity as a National Science Foundation Postdoctoral Fellow where he became the primary developer of the Chemistry and Harvard Macromolecular Mechanics (CHARMM) software system, which is useful in simulating motion and evaluating energies of macromolecular systems. In 1985, Dr. Brooks joined the staff of the Division of Computer Research and Technology at the National Institutes of Health where he became the Chief of the Molecular Graphics and Simulation Section of the Laboratory of Structural Biology. Dr. Brooks is currently the Chief of the Computational Biophysics Section of the Laboratory of Biophysical Chemistry (LBC) at the National Heart, Lung, and Blood Institute (NHLBI) where he continues to develop new methods and to apply these methods to both basic and specific problems of biomedical interest.

Yan A. Su is the Associate Professor in the Department of Pathology and a member in Cardinal Bernardin Cancer Center, Loyola University Medical Center at Chicago. He received his M.D. degree in Lanzhou Medical College and Ph.D. degree in University of Michigan. He had the postdoctoral training in both of Michigan Comprehensive Cancer Center, University of Michigan, and the National Human Genome Research

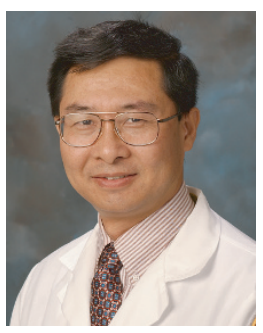
Institute, National Institutes of Health. Dr. Su was an Assistant Professor at Lombardi Cancer Center, Georgetown University Medical Center in 1997 and became an Associate Professor at Loyola University Chicago in 2002. His research effort focuses on molecular biology of malignant melanoma and breast cancer and he has the $\mathrm{NIH}$ funded projects in high-throughput analysis of gene expression. In addition, he is a member in the NIH study sections. 